\title{
STABILITY ANALYSIS IN THE FRAMEWORK OF DECISION MAKING UNDER RISK AND UNCERTAINTY
}

\author{
Dmitry Ivanov ${ }^{1}$, Alexander Arkhipov $^{2}$, Vera Tolkacheva ${ }^{2}$, Boris Sokolov $^{3}$ \\ ${ }^{\prime}$ Chemnitz University of Technology, GERMANY \\ dmitri.ivanov@mail.ru \\ ${ }^{2}$ Saint Petersburg State University of Technology and Design, RUSSIA \\ A_arkhipov@sutd.ru \\ vera_tolkacheva@mail.ru \\ ${ }^{3}$ Russian Academy of Science,
}

Saint Petersburg Institute of Informatics and Automation (SPIIRAS), RUSSIA

sokol@iias.spb.su

\begin{abstract}
Efficient methods of collaborative networks $(C N)$ configuration should provide models and algorithms of decision-making under risk and uncertainty. In this paper, we present a multi-disciplinary framework of decision making in $C N$. Particular feature of this framework is stability analysis. We analyse basics of stability analysis and its challenges in the $C N$ settings. Then we present a conceptual model of $\mathrm{CN}$ stability analysis and its dynamical interpretation. The stability analysis can be considered as an efficient tool to improve the quality of CN planning and execution models.
\end{abstract}

\section{INTRODUCTION}

In this paper, problem of value chain configuration and multi-echelon planning in collaborative networks $(\mathrm{CN})$ is addressed. One of the $\mathrm{CN}$ management challenges is a combined formation of the $\mathrm{CN}$ structural-functional-informational configuration and the programs of the $\mathrm{CN}$ execution. An important point of such simultaneous formation consists in ensuring of the business-processes continuity, information availability, and system stability.

A number of recent research papers (Camarihna-Matos, 2004, 2005) have dealt with forming of $\mathrm{CN}$ configuration methodology. The papers presented grounded models and algorithms of how to configure $\mathrm{CN}$ based on the known "normative" values of parameters. However, the $\mathrm{CN}$ execution is accomplished by permanent changes of internal network properties and external environment. So, the "normative" values of parameters can oscillate. As a consequence of this, (i) the analysis of $\mathrm{CN}$ stability and (ii) the embedding uncertainty factors into the planning models are needed. The second means that all the $\mathrm{CN}$ management phases (planning, monitor- 
ing, analysis, and adjustment) must be considered as a whole based on the unified methodological basis (Ivanov et al., 2006). The planning subjects must be not only original objects, but also dynamics of their interactions, environment, and models. Planning process is interpreted as continuous control of system dynamics under the terms of uncertainty. Results of planning are not only ideal operations model, but also a set of the $C N$ execution scenarios, models, algorithms, intended for system functioning support in case of disturbances and deviations. The above-mentioned challenges of the planning and control models interconnection are implemented in the DIMA-methodology (Ivanov, 2006). In this paper, we deal with the analysis of CN stability.

\section{A FRAMEWORK OF DECISION MAKING FOR THE CN CONFIGURATION}

We propose a framework of decision making for the $\mathrm{CN}$ configuration, which makes it possible to consider static network configuration and dynamic network reconfiguration combined. This framework is based on the combination of classic system and control theory with modern evolution system theories. The process of decision making is constructed as a combination of agent-based techniques with techniques of control and systems theories. It allows taking into account activity of the system elements, systematically risk embedding into the $\mathrm{CN}$ configuration and execution models, multiple model network description, interconnecting of configuration and execution processes.

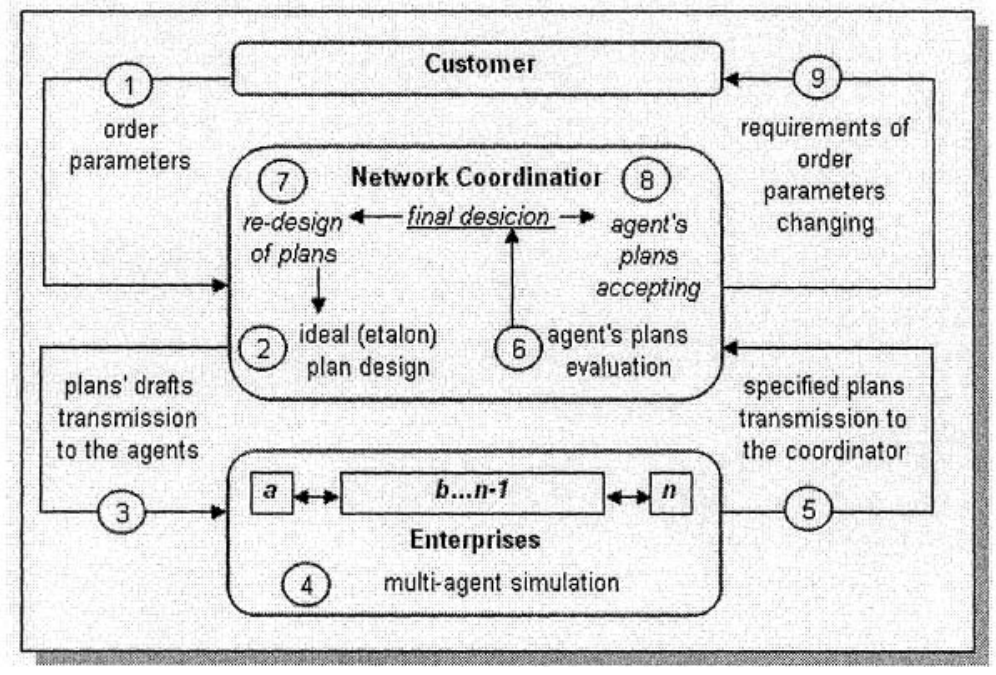

Figure 1. Decision making in the $\mathrm{CN}$ based on the combined using of multiagent and control theory frameworks

This framework is based on the DIMA-methodology (Ivanov, 2006). In the DIMA-methodology, the conventional modelling approaches are not set off with each other, but considered as a united integrated modelling framework. The multi- 
agent ideology is considered as a basis for the active elements modelling at the conceptual, mathematical, and simulation levels. The control theory serves as a theoretical background of systems analysis and synthesis (Casti, 1979, Sokolov and Yusupov, 2004). Figure 1 presents the process of decision making in the $\mathrm{CN}$ based on the combined using of multi-agent and control theory frameworks.

Algorithms of the control theory and operation research are intended for the ideal (etalon) solutions finding, which can be taken as a basis for the evaluation of the solutions found by agents in regard to their quality, fullness, and reliability. The second advantage consists in balancing the global network criteria with the local agents' criteria. Ideal (etalon) plans generated by the network coordinator are adjusted and specified by agents' interactions. So such a combined procedure of decision-making allows implementing decentralized management concept.

\section{BASICS AND CHALLENGES OF STABILITY ANALYSIS}

The degree of deviation in a functioning complex system (including $\mathrm{CN}$ ) from its planned states is related to the concepts of stability and sensitivity (Yusupov and Rozenwasser, 1999, Sterman, 2000). Sensitivity characterizes the rate of system's reaction in response to disturbances of different classes. Sensitivity analysis permits the determenation of potentially dangerous situations resulting from critical variations of system's functioning. Stability expresses the ability of a system to return to the initial (planned) state and (or) to remain within bounds of operation under the presence of perturbation factors. The stability analysis is performed regarding all of the states $S_{i}$ that lie within the control horizon considered. This is an important part of the $\mathrm{CN}$ operative diagnosis and forecasting. The methodology of the $\mathrm{CN}$ comprehensive tactical decision making and operative diagnosis and forecasting taking into account decision stability is a subject of our research field.

The concept of 'stability' plays a fundamental role in the systems theory. The sense of this term in general is equal for different types and classes of systems. It consists in limited reaction of a system on scale-limited entering impacts (controlled and non-controlled). A special feature of the $\mathrm{CN}$ stability analysis consists in adjustment of actions elaborated by managers and combination of centralized and decentralized management. This means that in case a $\mathrm{CN}$ looses its balance state, a search of new balance state is executed with the decentralized coordination of all $\mathrm{CN}$ participants' interests in the framework of common global criteria.

The $\mathrm{CN}$ differs from a physical system. The latter is remarkable for its planning mechanisms, which have some elements of subjectivism. That is why it becomes necessary to broaden the sense of 'stability' term while $\mathrm{CN}$ considering. $\mathrm{ACN}$ is stable in its planned state, if i) it has a fixed variety of possible adjustment actions; ii) scale-limited and low-power influences occur; iii) these influences cause scalelimited and low-power oscillation of end parameters of the $\mathrm{CN}$.

Let us examine some other aspects of the $\mathrm{CN}$ stability analysis. Stability characterizes a capability of a system to return to the initial (planned) state or to stay a certain period of time within admissible functioning area under the pressure of different disturbance factors. Moreover it is essential to understand, that the stability 
of a system is defined according to certain classes of disturbances. One of the aspects of stability analysis is the $\mathrm{CN}$ oscillation analysis. Usually three main classes of oscillations are introduced: damped oscillations, expanded oscillations, and chaotic oscillations (Sterman, 2000). The evaluation of the CN stability is meant for the final decision making about the network design, and is the last step in the network design process. The stability analysis is also necessary while $\mathrm{CN}$ functioning (in dynamics). The CN stability analysis is carried out within a certain period of time, because an influence of disturbance factors and their impact on the $\mathrm{CN}$ functioning have definite time delays.

\section{CONCEPTUAL MODEL OF STABILITY ANALYSIS FOR THE CN CONFIGURATION}

The stability analysis of the $\mathrm{CN}$ configuration is based on the conceptual model of $\mathrm{CN}$ design and control (Ivanov et al., 2006). After a set of non-dominated Pareto optimal alternative of admissible $\mathrm{CN}$ configurations is formed, the stability analysis takes place. In the $\mathrm{CN}$ settings, the stability analysis has two main particular features, namely (i) decentralization resulted in need of balancing global network criteria and local agents' criteria and (ii) existence of a number of alternative $C N$ configurations resulted in need of advancing stability analysis because of possibilities of structural-functional $\mathrm{CN}$ reconfiguration in dynamics. The conceptual model of the stability analysis can be presented in graphical form (Figure 2).

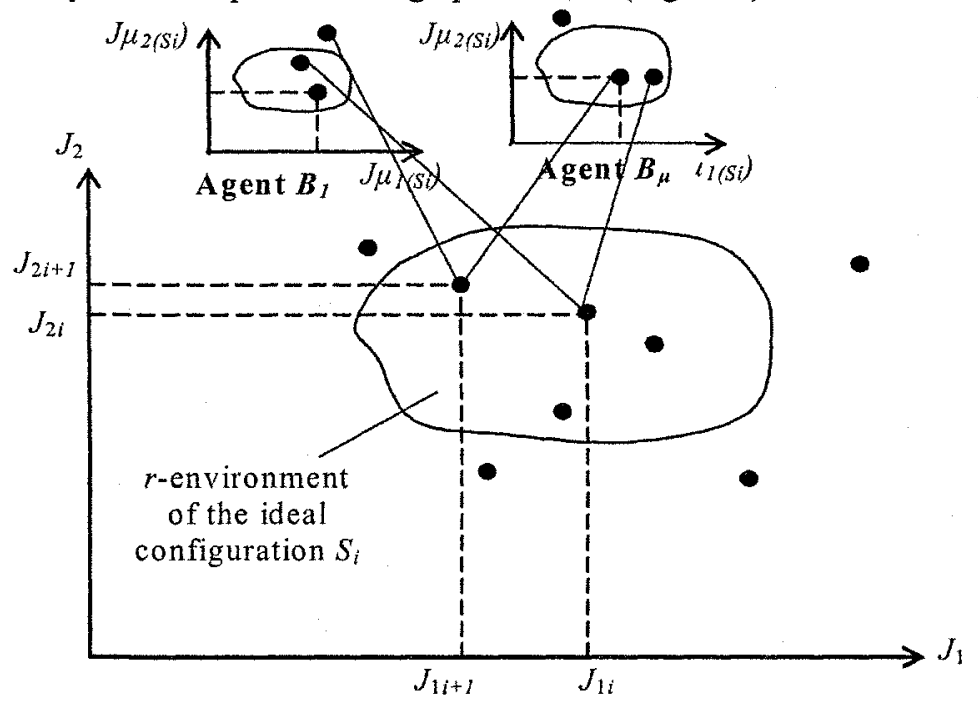

Figure 2. Conceptual model of the stability analysis

Figure 2 presents conceptual model of the stability analysis for a $\mathrm{CN}$ configuration $S_{i}$. To simplify the picture, we consider only two criteria $J_{1}$ and $J_{2}$ of $\mathrm{CN}$ configuration parameters

$$
\boldsymbol{J}(\boldsymbol{x}(t), \boldsymbol{u}(t), \boldsymbol{\xi}(t), t)=\left\|J_{1}, J_{2}, J_{3}, J_{4}, J_{5}, J_{6}, J_{7}, J_{8}\right\|^{\mathrm{T}},
$$


where $\boldsymbol{J}$ is a set of $\mathrm{CN}$ configuration parameters, $\boldsymbol{x}(t)$ is an initial state of the $\mathrm{CN}$, $\boldsymbol{u}(t)$ is a $\mathrm{CN}$ execution plan, and $\boldsymbol{\xi}(t), t)$ are vector functions that defines the minimum and maximum values of perturbation impacts. The point $\left(J_{2 i}, J_{l i}\right)$ corresponds with the ideal $\mathrm{CN}$ execution plan. Then we introduce the $r$-environment of the ideal configuration $S_{i}$, which borders permissible oscillations of the criteria $J_{1}$ and $J_{2}$, so that the $\mathrm{CN}$ goal criteria can be achieved.

In accordance with the framework of decision making in $\mathrm{CN}$ (see paragraph 2) and due to decentralization and structure dynamics, balancing global network criteria and local agents' criteria and stability checking of alternative $\mathrm{CN}$ configurations are needed. Each agent builds its $r$-environment on the above-described principles. The $\mathrm{CN}$ configuration $S_{i}$ can be selected and launched only if all agent plans are stable for each state of the given $\mathrm{CN}$ configuration within the $\mathrm{CN} r$-environment. Figure 2 presents an example when agent plans are stable regarding point $\left(J_{2 i}, J_{1 i}\right)$, but the plan of agent $B_{1}$ is instable regarding point $\left(J_{2 i+1}, J_{I i+1}\right)$. So the CN configuration $S_{i}$ can not be selected and launched, and further decision making procedures are needed.

The stability analysis objective at the configuration stage consists in indicating of permissible $\mathrm{CN}$ execution parameter oscillations. Based on the stability analysis results, the decision maker can estimate the stability degree of the configured $\mathrm{CN}$. As a rule, border extension of permissible $\mathrm{CN}$ execution parameter oscillations leads to worsened goal criteria values. The decision maker can simulate various $\mathrm{CN}$ configurations and execution scenarios trying to balance the goal criteria and the probability of their achieving. Additionally, other techniques of extended stability analysis such as $\mathrm{CN}$ stability reserve analysis and perceptivity analysis of $\mathrm{CN}$ execution parameters regarding various perturbation impacts can be applied.

The presented conceptual model is based on the forecasted information about the $\mathrm{CN}$ execution. However, the problem of including information update (Sethi et al., 2005) in $\mathrm{CN}$ decisions remains open. That is why the techniques of stability analysis in dynamics must be elaborated.

\section{STABILITY ANALYSIS IN DYNAMICS}

In dynamics, stability estimation can be performed on the basis of the attainability sets $D\left(t, T_{0}, X_{0}\right)$, where $X_{0}$ is a set of possible initial states of the system. To perform such analysis internal $D^{-}\left(t, T_{0}, X_{0}\right)$ and external $D^{+}\left(t, T_{0}, X_{0}\right)$ approximations of $D(t$, $T_{0}, X_{0}$ ) should be constructed. Let us suppose that a set of admissible disturbances $\Xi(x(t), t)$ is defined as follows:

$$
\xi_{j}^{(1)}(t) \leq \xi_{j}(t) \leq \xi_{j}^{(2)}(t), j=1, \ldots, m,
$$

where $\xi_{j}^{(1)}, \xi_{j}^{(2)}$ are vector functions for minimal and maximal disturbances consecutively. These disturbances may appear at the stage of each $\mathrm{CN}$ plan execution $\left(\boldsymbol{u}_{i}(t), t \in\left(T_{0}, T_{j}\right], i=1, \ldots, n\right)$ within some particular scenario of external environment influences on the $\mathrm{CN}\left(\boldsymbol{\xi}_{j}(t), t \in\left(T_{0}, T_{f}\right], j=1, \ldots, m\right)$. Let the initial $\mathrm{CN}$ state be $\boldsymbol{x}\left(T_{0}\right)$, hence examination of its functioning plan $\boldsymbol{u}_{i}(t)$ is needed. So, the defined vectors and disturbances space (2) for the scenario $\boldsymbol{\xi}_{j}(t)$ are corresponded to the area of 
possible variables of the $\mathrm{CN}$ configuration models, i.e. the set of different execution scenarios.

We propose this area to be named as the attainability set of the $\mathrm{CN}$ under disturbances. We define it as follows:

$$
D_{x}^{(\xi)}\left(T_{f}, T_{0}, X_{0}, \Xi, \boldsymbol{u}_{i}\right)
$$

The set $D_{x}^{(\xi)}\left(T_{f}, T_{0}, X_{0}, \Xi, \boldsymbol{u}_{i}\right)$ is corresponded to the indicators values, which assess the $\mathrm{CN}$ efficiency and stability. The latter we define as follows:

$$
D_{J}^{(\xi)}\left(T_{f}, T_{0}, X_{0}, \Xi, \boldsymbol{u}_{i}\right)
$$

To make the further material more comprehensive we will examine two components of vector index only. These components correspond to the indicators of effectiveness $\left(J_{1}\right)$ and resource-containing $\left(J_{2}\right)$ of the $\mathrm{CN}$ functioning. In this case while geometrically describing the attainability area, it becomes possible to use Decart system of coordinates instead of polar system.

If for some fixed functioning plans $\boldsymbol{u}_{i}(t),(i=1, \ldots, n)$ under disturbances $\xi_{j}(t)$ the requirements $(5)$ are fulfilled

$$
D_{J}^{(\xi)}\left(T_{f}, T_{0}, X_{0}, \Xi, \boldsymbol{u}_{i}\right) \subset P_{J}
$$

the $\boldsymbol{u}_{i}(t)$ management program (the plan of the $\mathrm{CN}$ functioning) is considered to be stable under disturbances $\xi_{i}(t)$. In other words, feasible $J_{1}, J_{2}$ deviations of quality indices of the $\mathrm{CN}$ functioning are considered to be acceptable.

In this case, the final selection of most stable $\mathrm{CN}$ management programs (plans of the $\mathrm{CN}$ implementation) is reasonable to be carried out accordingly to the following condition:

$$
S_{i}^{*}\left(\boldsymbol{u}_{i}(t)\right)=\max _{1 \leq i \leq n} \min _{1 \leq j \leq m} S_{i}\left(\boldsymbol{u}_{i}(t)\right),
$$

where $S_{i}\left(\boldsymbol{u}_{i}(t)\right)$ - is the area of sets $\overline{\bar{D}}_{J}^{(\xi)}\left(T_{f}, T_{0}, X_{0}, \Xi, \boldsymbol{u}_{i}\right)$ and $P_{J}$ intersection; $n$ - the total amount of analyzed CN implementation plans; $m$ - the total amount of disturbance scenarios at the stage of $\mathrm{CN}$ plan realization.

It is possible to show that the search of the most stable $\mathrm{CN}$ functioning plan due to statement (6) is the realization of the multi-criteria selection under uncertainty, i.e. the principle of the guaranteed result.

Finally, we consider the decentralized stability analysis. Dynamic system of the $\mathrm{CN}$ execution can be described in the following way:

$$
\begin{aligned}
& \dot{\vec{x}}_{\mu}=\vec{f}_{\mu}\left(\vec{x}_{\mu}, \vec{u}_{\mu}, t\right), \\
& \vec{u}_{\mu}(t) \in Q\left(\vec{x}_{\mu}, t\right), \\
& \vec{x}_{\mu}\left(T_{0}\right)=x_{\mu 0} ; \vec{x}_{\mu}\left(T_{f}\right) \in \mathrm{R}^{\tilde{n}^{\prime}}, \\
& J_{\mu}^{o b}=J_{\mu}^{o b}\left(\vec{x}_{1}\left(T_{f}\right), \vec{x}_{2}\left(T_{f}\right), \ldots, \vec{x}_{\tilde{m}^{\prime}}\left(T_{f}\right)\right),
\end{aligned}
$$

where $\vec{x}$ is a general $\mathrm{CN}$ state vector; $\vec{u}$ is a general $\mathrm{CN}$ control vector. The known vector-functions $\vec{h}_{0}$ and $\vec{h}_{1}$ determine end conditions for $\vec{x}$ at time $t=T_{0}$ and at 
$t=T_{f}$. The known vector-functions $\vec{q}^{(1)}(\vec{x}, \vec{u})=\vec{O}$ and $\vec{q}^{(2)}(\vec{x}, \vec{u}) \leq \vec{O}$ set main constraints for the agent operation, $\mu=1, \ldots, \widetilde{m}^{\prime}$ is a number of agents (local decision makers) performing control functions.

Now the stable plan construction for full decentralization can be stated as search for equilibrium program controls of $B_{1}, B_{2}, \ldots, B_{\tilde{m}^{\prime}}$ agents in a differential game. First, the attainability sets $D\left(T_{f}, T_{0}, \vec{x}_{\mu}\left(T_{0}\right)\right)$ for each agent $B_{1}, B_{2}, \ldots, B_{\widetilde{m}^{\prime}}$ at time $t=T_{f}$ is constructed. Then an equilibrium point for the system of functions $\left\{J_{\mu}^{o b}\left(x_{1}\left(T_{f}\right), x_{2}\left(T_{f}\right), \ldots, x_{\vec{m}^{\prime}}\left(T_{f}\right)\right)\right\}$ over $D\left(T_{f}, T_{0}, \vec{x}_{1}\left(T_{0}\right)\right) \times D\left(T_{f}, T_{0}, \vec{x}_{2}\left(T_{0}\right)\right) \times \ldots$ $\times D\left(T_{f}, T_{0}, \vec{x}_{\widetilde{m}^{\prime}}\left(T_{0}\right)\right)$ can be determined. Here Brown-Robinson's method and its modifications can be used. After that management actions $\vec{u}_{\mu}(t), t \in\left(T_{0}, T_{f}\right]$ for transition from the initial state $\vec{x}_{\mu}\left(T_{0}\right)=\vec{x}_{\mu 0}$ to the final state $\vec{x}_{\mu}\left(T_{f}\right)=\vec{x}_{\mu}^{(p c)}$ are constructed.

The set of agents $B_{\mu}, \mu=1, \ldots, \widetilde{m}^{\prime}$ is split into the following subsets $\chi_{\zeta}, \zeta=1, \ldots, \mathrm{P} ; \chi_{\zeta} \cap \chi_{\zeta^{\prime}}=\varnothing, \zeta \neq \zeta^{\prime}, \bigcup_{\zeta=1}^{\mathrm{P}} \chi_{\zeta}=\chi, \chi=\left\{\chi_{1}, \ldots, \chi_{\mathrm{P}}\right\}, \chi \subseteq \mathrm{B}(\mathrm{M}), \mathrm{M}=\left\{1, \ldots, \widetilde{m}^{\prime}\right\}$.

There is a subset for each coalition (a group of agents $B_{\mu}$ united into a competence structure). The set $\chi$ is called a coalition structure. We have examined three variants of coalition communication, namely (i) if agents of the coalition $\chi_{\zeta}$ do not know coalition structure $\chi$ or functioning modes of other coalitions $\chi_{\zeta^{\prime}}$ (where $\zeta$, $\left.\zeta^{\prime} \in\{1, \ldots, P\}\right)$ in coalition structure $\chi$, (ii) if agents in coalition $\chi_{\zeta}$ know the coalition structure $\chi$, and (iii) if a finite set $\mathfrak{R}$ of coalition structures is known, rather than a particular structure $\chi$.

\section{CONCLUSIONS}

Decision making techniques for the $\mathrm{CN}$ configuration must take into account risk and uncertainty as well as support decision making in decentralized way. In this paper, we presented a multi-disciplinary framework of decision making in $\mathrm{CN}$, based on combination of control theory and multi-agent approach. Particular feature of this framework is stability analysis. We analysed basics of stability analysis and revealed its challenges in the $\mathrm{CN}$ settings. Then we presented a conceptual model of $\mathrm{CN}$ stability analysis and its dynamical interpretation. The stability analysis objective consists in indicating of permissible $\mathrm{CN}$ execution parameter oscillations. As a rule, border extension of permissible $\mathrm{CN}$ execution parameter oscillations leads to worsened goal criteria values. So, the stability analysis provides efficient tool how to balance the goal criteria and the probability of their achieving. The conceptual model of stability analysis is based on the forecasted information about the $\mathrm{CN}$ execution. The technique of stability analysis in dynamics includes information 
update in $\mathrm{CN}$ decisions and provides their adequacy to the current execution environment.

The presented stability analysis technique provides efficient tool how to embed risk and uncertainty factors into the $\mathrm{CN}$ configuration and execution models. Development of CN stability analysis methods has practical and theoretical importance. Generally, the efforts of $\mathrm{CN}$ configuration (scheduling) algorithms improving can be meaningful only in combination with appropriate $\mathrm{CN}$ stability analysis methods. Stability may be regarded as an additional indicator for the CN analysis, modeling, planning, operative management and forecasting. Its application in the $\mathrm{CN}$ design and control models not only supports theoretical basis of the $\mathrm{CN}$, but has a practical importance also. It may be applied to improve quality and precision of planning and management, decision taking (at levels of the $\mathrm{CN}$ goal selection, scheduling, monitoring, forecasting and adjustment), as well as complex analysis of the $\mathrm{CN}$ activity, forecasting and strategic decisions elaboration.

\section{ACKNOLEDMENTS}

The research described in this paper is partially supported by grants from Russian Foundation for Basic Research (grant №05-07-90088), Institute for System Analysis RAS (Project 2.5), CRDF Project \#: Rum2-1554-ST-05, and the Alexander von Humboldt Foundation.

\section{REFERENCES}

1. Camarinha-Matos, L. (ed.) (2004). Virtual Enterprises and Collaborative Networks, Kluwer Academic Publishers.

2. Camarinha-Matos, L., Afsarmanesh, H. and A. Ortiz (eds.) (2005). Collaborative Networks and Their Breeding Environments, Springer.

3. Casti JL. Connectivity, Complexity and Catastrophe in Large-Scale Systems. Wiley-Intersc., 1979.

4. Ivanov D., Arkhipov A., Sokolov B.: Intelligent Supply Chain Planning in Virtual Enterprises. In: Virtual Enterprises and Collaborative Networks, edited by L.Camarihna-Matos, Kluwer Academic Publishers, 2004: 215-223.

5. Ivanov, D., Käschel, J., Arkhipov, A., Sokolov, B., and Zschorn L. (2005): Quantitative Models of Collaborative Networks, In: Collaborative Networks and Their Breeding Environments, edited by L.Camarihna-Matos, H. Afsarmanesh, A. Ortiz, Springer, 2005, pp. 387-394.

6. Ivanov, D.A., Arkhipov, A.V., and Sokolov, B.V. (2006): Intelligent planning and control of manufacturing supply chains in virtual enterprises, in: International Journal of Manufacturing Technology and Management, in print.

7. Ivanov, D.A. (2006). DIMA - Decentralized Integrated Modeling Approach - Towards The Enterprise Network Theory, Proceedings of the German-Russian Logistics Workshop, 20.-21 April, Saint Petersburg, Russia, 2006, pp. 23-46.

8. Sethi, SP, Yan, H., Zhang, $H$. Inventory and Supply Chain Management with Forecast Updates. Springer, 2005.

9. Sokolov BV, Yusupov RM. Conceptual Foundations of Quality Estimation and Analysis for Models and Multiple-Model Systems. Int. Journal of Computer and System Sciences, 6(2004): 5-16.

10. Sterman JD. Business dynamics: systems thinking and modeling. McGraw-Hill, 2000. 